\title{
Multi gluon correlations in the Color Glass \\ Condensate: quantum interference in proton-nucleus collisions
}

\author{
Tolga Altinoluk \\ National Centre for Nuclear Research, 00-681 Warsaw, Poland \\ E-mail: tolga.altinoluk@ncbj.gov.pl
}

\section{Néstor Armesto*}

Instituto Galego de Física de Altas Enerxías IGFAE,

Universidade de Santiago de Compostela, 15782 Santiago de Compostela, Galicia-Spain

E-mail: nestor.armesto@usc.es

\author{
Alex Kovner \\ Physics Department, University of Connecticut, 2152 Hillside Road, Storrs, CT 06269, USA \\ E-mail: kovner@phys. uconn. edu \\ Michael Lublinsky \\ Physics Department, Ben-Gurion University of the Negev, Beer Sheva 84105, Israel \\ E-mail: lublinm@bgu.ac.il
}

\begin{abstract}
We compute multi gluon production at mid rapidity in proton-nucleus collisions within the Color Glass Condensate framework. We show that, in the dilute-dense limit valid for such collisions, the terms responsible for the multi gluon correlation have two origins. On the one hand, the HanburyBrown-Twiss interference in the final state. On the other hand, the Bose enhancement of gluons in the projectile and target wave functions, with the latter being suppressed by the number of colors with respect to the former. We also demonstrate that such correlations come from the highest order relevant correlator of Wilson lines in the target wave function, i.e., the quadrupole and sextupole for two and three gluon correlations respectively. We develop a general method for the computation of such high order correlators that captures the bulk of their contribution to the multi gluon production cross section but does not employ the approximation of a large number of colours.
\end{abstract}

International Conference on Hard and Electromagnetic Probes of High-Energy Nuclear Collisions 30 September - 5 October 2018

Aix-Les-Bains, Savoie, France

\footnotetext{
* Speaker.
} 


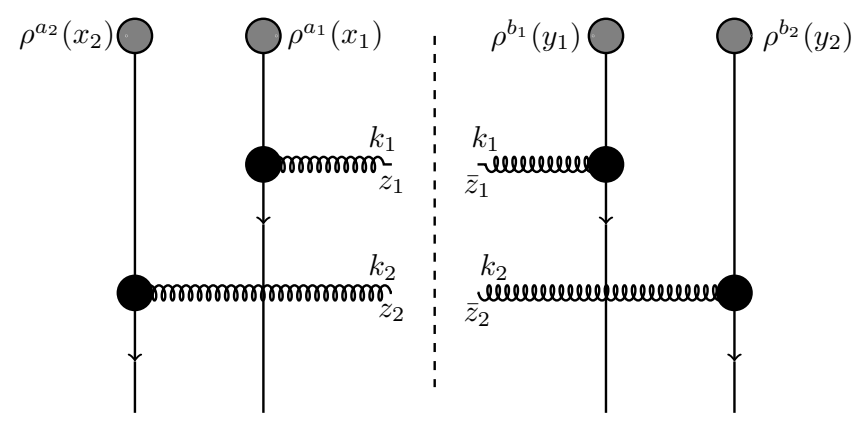

Figure 1: Graphical illustration of Eq. (2.1) with the vertical lines representing the rescatterings with the target through Wilson lines.

\section{Introduction}

The measurement of two particle correlations extended in pseudorapidity and peaked when the particles are parallel or antiparallel in azimuth - the ridge - in pp and $\mathrm{pPb}$ collisions at the LHC and $\mathrm{dAu}$ at RHIC, together with the finding of the striking similarities with nucleus-nucleus in this and many other observables usually considered as signatures of the existence of deconfined quark-gluon matter, has triggered a large theoretical and experimental activity on the subject, see [1]. While the standard explanation of such azimuthal correlations in nucleus-nucleus collisions are final state interactions leading to the applicability of relativistic hydrodynamics, it seems less justified in smaller systems and initial state approaches have also been essayed. Among them, those based on the Color Glass Condensate CGC seem to be the most promising for a first principle explanation of such phenomenon. CGC explanations have been successful [2] in describing the ridge in pp collisions under the 'Glasma graphs' approximation [3] valid for collisions between dilute systems.

We have shown in previous works [4] that the correlations in the Glasma graph approach have two origins: Bose enhancement (BE) of the gluons in the projectile and target wave function (WF), and Hanbury-Brown-Twiss (HBT) correlations of the final state gluons. In this contribution we summarise the findings in [5] where an extension of the CGC approach to dilute-dense collisions (see also [6]) has been obtained and applied to two and three gluon correlations. We focus here only in deriving the correlations giving rise to even harmonics, for the problem of odd harmonics and the comparison to experimental data see [1].

\section{Two gluon correlations}

In the CGC, the two gluon inclusive cross section (graphically shown in Fig. 1) is given by

$$
\begin{aligned}
& \frac{d \sigma}{d^{2} k_{1} d \eta_{1} d^{2} k_{2} d \eta_{2}}=\alpha_{s}^{2}(4 \pi)^{2} \int_{z_{1} \bar{z}_{1} z_{2} \bar{z}_{2}} e^{i k_{1} \cdot\left(z_{1}-\bar{z}_{1}\right)+i k_{2} \cdot\left(z_{2}-\bar{z}_{2}\right)} \\
\times & \int_{x_{1} x_{2} y_{1} y_{2}} A^{i}\left(x_{1}-z_{1}\right) A^{i}\left(\bar{z}_{1}-y_{1}\right) A^{j}\left(x_{2}-z_{2}\right) A^{j}\left(\bar{z}_{2}-y_{2}\right)\left\langle\rho^{a_{1}}\left(x_{1}\right) \rho^{a_{2}}\left(x_{2}\right) \rho^{b_{1}}\left(y_{1}\right) \rho^{b_{2}}\left(y_{2}\right)\right\rangle_{P} \\
\times & \left\langle\left[U\left(z_{1}\right)-U\left(x_{1}\right)\right]^{a_{1} c}\left[U^{\dagger}\left(\bar{z}_{1}\right)-U^{\dagger}\left(y_{1}\right)\right]^{c b_{1}}\left[U\left(z_{2}\right)-U\left(x_{2}\right)\right]^{a_{2} d}\left[U^{\dagger}\left(\bar{z}_{2}\right)-U^{\dagger}\left(y_{2}\right)\right]^{d b_{2}}\right\rangle_{T},
\end{aligned}
$$


with $\int_{z} \equiv \int d^{2} z, U(x)$ the adjoint Wilson line at transverse position $x$ and

$$
A^{i}(x-y)=-\frac{1}{2 \pi} \frac{(x-y)_{i}}{(x-y)^{2}}=\int \frac{d^{2} k}{(2 \pi)^{2}} e^{-i k \cdot(x-y)} \frac{k^{i}}{k^{2}} .
$$

$\langle\cdots\rangle_{P, T}$ denote the averages on the projectile and target wave function of the projectile sources and Wilson lines describing the rescattering with the target, respectively.

For the dilute projectile average, we consider pairwise contractions

$$
\begin{array}{r}
\left\langle\rho^{a_{1}}\left(x_{1}\right) \rho^{a_{2}}\left(x_{2}\right) \rho^{b_{1}}\left(y_{1}\right) \rho^{b_{2}}\left(y_{2}\right)\right\rangle_{P}=\left\langle\rho^{a_{1}}\left(x_{1}\right) \rho^{a_{2}}\left(x_{2}\right)\right\rangle_{P}\left\langle\rho^{b_{1}}\left(y_{1}\right) \rho^{b_{2}}\left(y_{2}\right)\right\rangle_{P} \\
+\left\langle\rho^{a_{1}}\left(x_{1}\right) \rho^{b_{1}}\left(y_{1}\right)\right\rangle_{P}\left\langle\rho^{a_{2}}\left(x_{2}\right) \rho^{b_{2}}\left(y_{2}\right)\right\rangle_{P}+\left\langle\rho^{a_{1}}\left(x_{1}\right) \rho^{b_{2}}\left(y_{2}\right)\right\rangle_{P}\left\langle\rho^{a_{2}}\left(x_{2}\right) \rho^{b_{1}}\left(y_{1}\right)\right\rangle_{P},
\end{array}
$$

using a generalisation of the McLerran-Venugopalan model [7]

$$
\left\langle\rho^{a}(x) \rho^{b}(y)\right\rangle_{P}=\delta^{a b} \mu^{2}(x, y)
$$

that results in dipole-dipole and quadrupole target averages, where $\mu^{2}(x, y)$ is a distribution that falls rapidly for $|x+y|>R_{P}$ with $R_{P}$ the radius of the projectile describing the valence colour charges in the projectile. For performing the target averages, we note that the cross section (2.1) involves integration over all transverse coordinates, so the largest contribution comes from maximising the distances. In the Glasma graphs approximation, the Wilson lines are expanded to the lowest order in the target fields and then pairwise contractions are performed. We follow here a different strategy (see also [8, 9]), taking into account that the target ensemble must be colour neutral and that in the CGC colour neutralisation takes place at distances $1 / Q_{s}$ with $Q_{s}$ the saturation scale of the target, so the $S$-matrix becomes non zero for colour singlets of size $>1 / Q_{s}$. Therefore, the leading contribution (in powers of $R_{P} Q_{s}$ ) comes from the $2 n$ points in $\langle\cdots\rangle_{T}$ combined into $n$ singlets at distances as large as possible. We realise this idea defining

$$
\left\langle U^{a b}(x) U^{c d}(y)\right\rangle_{T}=\delta^{a c} \delta^{b d} \frac{1}{\left(N_{c}^{2}-1\right)^{2}}\left\langle\operatorname{tr}\left[U(x) U^{\dagger}(y)\right]\right\rangle_{T}=\delta^{a c} \delta^{b d} \frac{1}{N_{c}^{2}-1} d(x, y),
$$

where

$$
d(x, y) \equiv\langle D(x, y)\rangle_{T},
$$

so

$$
\begin{gathered}
\langle Q(x, y, z, v)\rangle_{T} \longrightarrow d(x, y) d(z, v)+d(x, v) d(z, y)+\frac{1}{N_{c}^{2}-1} d(x, z) d(y, v), \\
\langle D(x, y) D(z, v)\rangle_{T} \longrightarrow d(x, y) d(z, v)+\frac{1}{\left(N_{c}^{2}-1\right)^{2}}[d(x, v) d(y, z)+d(x, z) d(v, y)] .
\end{gathered}
$$

The final result for a translationally invariant target can be organised in powers of the number of colours $N_{c}$ :

$\frac{d \sigma}{d^{2} k_{1} d \eta_{1} d^{2} k_{2} d \eta_{2}}=\alpha_{s}^{2}(4 \pi)^{2}\left(N_{c}^{2}-1\right)^{2} \int \frac{d^{2} q_{1}}{(2 \pi)^{2}} \frac{d^{2} q_{2}}{(2 \pi)^{2}} d\left(q_{1}\right) d\left(q_{2}\right)\left\{I_{0}+\frac{1}{N_{c}^{2}-1} I_{1}+\frac{1}{\left(N_{c}^{2}-1\right)^{2}} I_{2}\right\}$,

with $I_{0}$ giving the uncorrelated emission of two gluons coming from the dipole-dipole terms, $I_{1}$ giving Bose enhancement of the projectile wave function and HBT coming from the quadrupole terms, and $I_{2}$ Bose enhancement in the target wave function coming from the dipole-dipole terms and $N_{c}$ corrections to projectile Bose and HBT coming from the quadrupole terms. 


\section{Three gluon correlations}

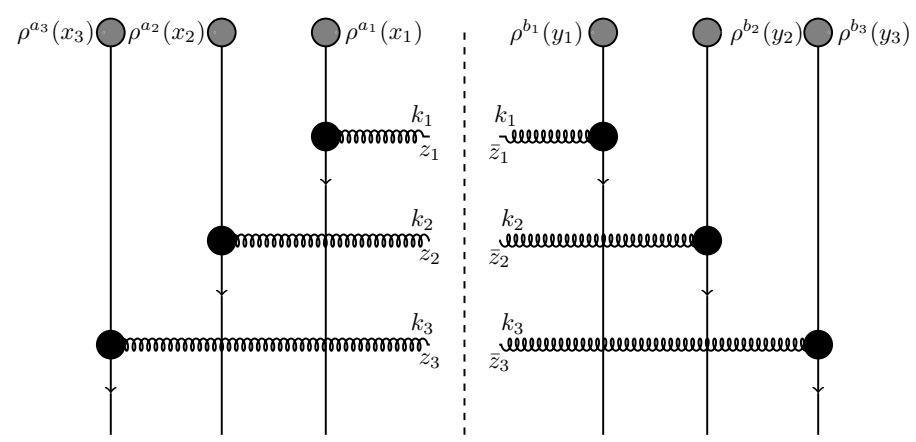

Figure 2: Graphical illustration of Eq. (3.1) with the vertical lines representing the rescatterings with the target through Wilson lines.

The three gluon inclusive cross section (graphically shown in Fig. 2) is given by

$$
\begin{aligned}
& \frac{d \sigma}{d^{2} k_{1} d \eta_{1} d^{2} k_{2} d \eta_{2} d^{2} k_{3} d \eta_{3}}=\alpha_{s}^{3}(4 \pi)^{3} \int_{z_{1} \bar{z}_{1} z_{2} \bar{z}_{2} z_{3} \bar{z}_{3}} e^{i k_{1} \cdot\left(z_{1}-\bar{z}_{1}\right)+i k_{2} \cdot\left(z_{2}-\bar{z}_{2}\right)+i k_{3} \cdot\left(z_{3}-\bar{z}_{3}\right)} \\
& \times \int_{x_{1} y_{1} x_{2} y_{2} x_{3} y_{3}} A^{i}\left(x_{1}-z_{1}\right) A^{i}\left(\bar{z}_{1}-y_{1}\right) A^{j}\left(x_{2}-z_{2}\right) A^{j}\left(\bar{z}_{2}-y_{2}\right) A^{k}\left(x_{3}-z_{3}\right) A^{k}\left(\bar{z}_{3}-y_{3}\right) \\
& \times\left\langle\rho^{a_{1}}\left(x_{1}\right) \rho^{a_{2}}\left(x_{2}\right) \rho^{a_{3}}\left(x_{3}\right) \rho^{b_{1}}\left(y_{1}\right) \rho^{b_{2}}\left(y_{2}\right) \rho^{b_{3}}\left(y_{3}\right)\right\rangle_{P} \\
& \times\left\langle\left(U_{z_{1}}-U_{x_{1}}\right)^{a_{1} c_{1}}\left(U_{\bar{z}_{1}}^{\dagger}-U_{y_{1}}^{\dagger}\right)^{c_{1} b_{1}}\left(U_{z_{2}}-U_{x_{2}}\right)^{a_{2} c_{2}}\left(U_{\bar{z}_{2}}^{\dagger}-U_{y_{2}}^{\dagger}\right)^{c_{2} b_{2}}\left(U_{z_{3}}-U_{x_{3}}\right)^{a_{3} c_{3}}\left(U_{\bar{z}_{3}}^{\dagger}-U_{y_{3}}^{\dagger}\right)^{c_{3} b_{3}}\right\rangle_{T} .
\end{aligned}
$$

Projectile averages done through pairwise contractions give rise to 15 terms which correspond to triple dipole (ddd), dipole-quadrupole (dQ) and sextupole (X) target averages. For these we follow the strategy explained in the previous Section which for the sextupole results in

$$
\begin{aligned}
& \left\langle\mathrm{X}\left(x_{1}, x_{1}^{\prime}, x_{2}, x_{2}^{\prime}, x_{3}, x_{3}^{\prime}\right)\right\rangle_{T} \longrightarrow d\left(x_{1}, x_{1}^{\prime}\right) d\left(x_{2}, x_{2}^{\prime}\right) d\left(x_{3}, x_{3}^{\prime}\right)+d\left(x_{1}, x_{3}^{\prime}\right) d\left(x_{2}, x_{1}^{\prime}\right) d\left(x_{3}, x_{2}^{\prime}\right) \\
& +d\left(x_{1}, x_{1}^{\prime}\right) d\left(x_{2}, x_{3}^{\prime}\right) d\left(x_{3}, x_{2}^{\prime}\right)+d\left(x_{2}, x_{2}^{\prime}\right) d\left(x_{3}, x_{1}^{\prime}\right) d\left(x_{1}, x_{3}^{\prime}\right)+d\left(x_{3}, x_{3}^{\prime}\right) d\left(x_{1}, x_{2}^{\prime}\right) d\left(x_{2}, x_{1}^{\prime}\right) \\
& +\frac{1}{N_{c}^{2}-1}\left\{d\left(x_{1}, x_{1}^{\prime}\right) d\left(x_{2}, x_{3}\right) d\left(x_{2}^{\prime}, x_{3}^{\prime}\right)+d\left(x_{2}, x_{2}^{\prime}\right) d\left(x_{3}, x_{1}\right) d\left(x_{3}^{\prime}, x_{1}^{\prime}\right)+d\left(x_{3}, x_{3}^{\prime}\right) d\left(x_{1}, x_{2}\right) d\left(x_{1}^{\prime}, x_{2}^{\prime}\right)\right. \\
& \left.\quad+d\left(x_{2}, x_{3}\right) d\left(x_{1}, x_{3}^{\prime}\right) d\left(x_{1}^{\prime}, x_{2}^{\prime}\right)+d\left(x_{3}, x_{1}\right) d\left(x_{2}, x_{1}^{\prime}\right) d\left(x_{2}^{\prime}, x_{3}^{\prime}\right)+d\left(x_{1}, x_{2}\right) d\left(x_{3}, x_{2}^{\prime}\right) d\left(x_{3}^{\prime}, x_{1}^{\prime}\right)\right\} \\
& +\frac{1}{\left(N_{c}^{2}-1\right)^{2}}\left\{d\left(x_{1}, x_{2}\right) d\left(x_{3}, x_{1}^{\prime}\right) d\left(x_{2}^{\prime}, x_{3}^{\prime}\right)+d\left(x_{2}, x_{3}\right) d\left(x_{1}, x_{2}^{\prime}\right) d\left(x_{3}^{\prime}, x_{1}^{\prime}\right)+d\left(x_{3}, x_{1}\right) d\left(x_{2}, x_{3}^{\prime}\right) d\left(x_{1}^{\prime}, x_{2}^{\prime}\right)\right. \\
& \left.\quad+d\left(x_{1}, x_{2}^{\prime}\right) d\left(x_{3}, x_{1}^{\prime}\right) d\left(x_{2}, x_{3}^{\prime}\right)\right\} .
\end{aligned}
$$

Again, the final result for a translationally invariant target can be organised in powers of $N_{c}$ :

$$
\begin{aligned}
& \frac{d \sigma}{d^{2} k_{1} d \eta_{1} d^{2} k_{2} d \eta_{2} d^{2} k_{3} d \eta_{3}}=\alpha_{s}^{3}(4 \pi)^{3}\left(N_{c}^{2}-1\right)^{3} \int \frac{d^{2} q_{1}}{(2 \pi)^{2}} \frac{d^{2} q_{2}}{(2 \pi)^{2}} \frac{d^{2} q_{3}}{(2 \pi)^{2}} d\left(q_{1}\right) d\left(q_{2}\right) d\left(q_{3}\right) \\
& \times\left\{I_{\mathrm{ddd}, 0}+\frac{1}{N_{c}^{2}-1}\left[I_{\mathrm{dQ}, 1}+I_{\mathrm{dQ}, 2}+I_{\mathrm{dQ}, 3}\right]+\frac{1}{\left(N_{c}^{2}-1\right)^{2}}\left(\left[I_{\mathrm{ddd}, 1}+I_{\mathrm{ddd}, 2}+I_{\mathrm{ddd}, 3}\right]\right.\right. \\
& \left.\left.+\left[I_{\mathrm{dQ}, 1}^{\prime}+I_{\mathrm{dQ}, 2}^{\prime}+I_{\mathrm{dQ}, 3}^{\prime}\right]+\left[I_{\mathrm{X}, 1}+I_{\mathrm{X}, 2}+I_{\mathrm{X}, 3}+I_{\mathrm{X}, 4}+I_{\mathrm{X}, 5}\right]\right)+\mathscr{O}\left[\left(N_{c}^{2}-1\right)^{-3}\right]+\mathscr{O}\left[\left(N_{c}^{2}-1\right)^{-4}\right]\right\} .
\end{aligned}
$$


This final result contains independent emission of 3 gluons $\left(I_{\mathrm{ddd}, 0}\right)$, independent emission of one gluon and projectile BE and HBT of the other two $\left(I_{\mathrm{dQ}, i}\right)$, independent emission of one gluon and target BE of the other two $\left(I_{\mathrm{ddd}, \mathrm{i}}\right), N_{c}$-suppressed independent emission of one gluon and projectile $\mathrm{BE}$ of the other two $\left(I_{\mathrm{dQ}, \mathrm{i}}^{\prime}\right)$, HBT of two gluons and projectile BE of other two $\left(I_{\mathrm{X}, 1,2,3}\right)$, and projectile BE $\left(I_{\mathrm{X}, 4}\right)$ and $\mathrm{HBT}\left(I_{\mathrm{X}, 5}\right)$ of the three gluons.

Summarising, by explicitly computing two and three gluon inclusive cross sections under the mentioned target averaging procedure, we find that the two main effects contributing to correlations, BE of the gluons in the projectile and target WFs, and HBT correlations of the final state gluons, survive density corrections in the target, that correlations between all particles come from the highest order Wilson line correlator, and that target BE correlations are suppressed by the number of colours with respect to projectile ones (at variance with what happens in the symmetric Glasma graphs approach where both are of the same order in $N_{c}$ ).

Acknowledgments: I thank the organisers for their invitation to provide this talk, A. Rezaeian and V. Skokov for discussions, and M. Martínez, M. Sievert and D. Wertepny for pointing out some mistakes in the first version of [5]. The work of TA is supported by Grant No. 2017/26/M/ST2/01074 of the National Science Centre, Poland. NA was supported by Ministerio de Ciencia e Innovación of Spain under projects FPA201458293-C2-1-P, FPA2017-83814-P and Unidad de Excelencia María de Maetzu under project MDM-20160692, by Xunta de Galicia (Consellería de Educación) within the Strategic Unit AGRUP2015/11, and by FEDER. AK was supported by the NSF Nuclear Theory grant 1614640. ML was supported by the Israeli Science Foundation grant \# 1635/16; AK and ML were also supported by the BSF grants \#2012124 and \#2014707. This work has been performed in the framework of COST Action CA15213 THOR.

\section{References}

[1] Talks by E. Chapon, M. Mace, A. H. Mueller, S. Schlichting and V. Skokov in this conference.

[2] K. Dusling and R. Venugopalan, Phys. Rev. Lett. 108 (2012) 262001 [arXiv:1201.2658 [hep-ph]]; Phys. Rev. D 87 (2013) no.5, 051502 [arXiv:1210.3890 [hep-ph]]; Phys. Rev. D 87 (2013) no.5, 054014 [arXiv:1211.3701 [hep-ph]]; Phys. Rev. D 87 (2013) no.9, 094034 [arXiv:1302.7018 [hep-ph]].

[3] A. Dumitru, F. Gelis, L. McLerran and R. Venugopalan, Nucl. Phys. A 810 (2008) 91 [arXiv:0804.3858 [hep-ph]]; A. Dumitru, K. Dusling, F. Gelis, J. Jalilian-Marian, T. Lappi and Venugopalan Phys. Lett. B697 (2011) 21 [arXiv:1009.5295].

[4] T. Altinoluk, N. Armesto, G. Beuf, A. Kovner and M. Lublinsky, Phys. Lett. B751 (2015) 448 [arXiv:1503.07126 [hep-ph]]; Phys. Lett. B752 (2016) 113 [arXiv:1509.03223 [hep-ph]].

[5] T. Altinoluk, N. Armesto, A. Kovner and M. Lublinsky, Eur. Phys. J. C 78 (2018) no.9, 702 [arXiv:1805.07739 [hep-ph]].

[6] T. Altinoluk, N. Armesto and D. E. Wertepny, JHEP 1805 (2018) 207 [arXiv:1804.02910 [hep-ph]].

[7] L. D. McLerran and R. Venugopalan, Phys. Rev. D 49 (1994) 2233 [hep-ph/9309289]; Phys. Rev. D 49 (1994) 3352 [hep-ph/9311205].

[8] Y. V. Kovchegov and D. E. Wertepny, Nucl. Phys. A906 (2013) 50 [arXiv:1212.1195] [hep-ph]; Nucl. Phys. A 925 (2014) 254 [arXiv:1310.6701 [hep-ph]].

[9] A. Kovner and A. Rezaeian, Phys.Rev. D97 (2018) 074008 [ arXiv:1801.04875 [hep-ph]] . 